\title{
Reinforcement Evolutionary Learning for Neuro-Fuzzy Controller Design
}

\author{
Cheng-Jian Lin ${ }^{+}$ \\ National University of Kaohsiung, Kaohsiung, \\ Taiwan
}

\section{Introduction}

In recent years, the concept of the fuzzy logic or artificial neural networks for control problems has grown into a popular research area [1]-[3]. The reason is that classical control theory usually requires a mathematical model for designing controllers. The inaccuracy of mathematical modeling of plants usually degrades the performance of the controllers, especially for nonlinear and complex control problems [4], [25]. Fuzzy logic has the ability to express the ambiguity of human thinking and translate expert knowledge into computable numerical data.

A fuzzy system consists of a set of fuzzy IF-THEN rules that describe the input-output mapping relationship of the networks. Obviously, it is difficult for human experts to examine all the input-output data from a complex system to find proper rules for a fuzzy system. To cope with this difficulty, several approaches that are used to generate the fuzzy IF-THEN rules from numerical data have been proposed [5]-[8]. These methods were developed for supervised learning; i.e., the correct "target" output values are given for each input pattern to guide the learning of the network. However, most of the supervised learning algorithms for neuro-fuzzy networks require precise training data to tune the networks for various applications. For some real world applications, precise training data are usually difficult and expensive, if not impossible, to obtain. For this reason, there has been a growing interest in reinforcement learning algorithms for use in fuzzy [9]-[10] or neural controller [11]-[12] design.

In the design of a fuzzy controller, adjusting the required parameters is important. To do this, back-propagation (BP) training was widely used in [11]-[12], [18]. It is a powerful training technique that can be applied to networks with a forward structure. Since the steepest descent technique is used in BP training to minimize the error function, the algorithms may reach the local minima very fast and never find the global solution.

The development of genetic algorithms (GAs) has provided another approach for adjusting parameters in the design of controllers. GA is a parallel and global technique [9], [19]. Because it simultaneously evaluates many points in a search space, it is more likely to converge toward the global solution. Some researchers have developed methods to design and implement fuzzy controllers by using GAs. Karr [2] used a GA to generate membership

+ Corresponding author. Email:chlin@nuk.edu.tw

Source: Reinforcement Learning: Theory and Applications, Book edited by Cornelius Weber, Mark Elshaw and Norbert Michael Mayer ISBN 978-3-902613-14-1, pp.424, January 2008, I-Tech Education and Publishing, Vienna, Austria 
functions for a fuzzy system. In Karr's work, a user needs to declare an exhaustive rule set and then use a GA to design only the membership functions. In [20], a fuzzy controller design method that used a GA to find the membership functions and the rule sets simultaneously was proposed. Lin [27] proposed a hybrid learning method which combines the GA and the least-squares estimate (LSE) method to construct a neuron-fuzzy controller. In [20] and [27], the input space was partitioned into a grid. The number of fuzzy rules (i.e., the length of each chromosome in the GA) increased exponentially as the dimension of the input space increased. To overcome this problem, Juang [26] adopted a flexible partition approach in the precondition part. The method has the admirable property of small network size and high learning accuracy.

Recently, some researchers [9], [19], [28]-[29] applied GA methods to implement reinforcement learning in the design of fuzzy controllers. Lin and Jou [9] proposed GAbased fuzzy reinforcement learning to control magnetic bearing systems. In [19], Juang and his colleagues proposed genetic reinforcement learning in designing fuzzy controllers. The GA adopted in [19] was based upon traditional symbiotic evolution which, when applied to fuzzy controller design, complements the local mapping property of a fuzzy rule. In [28], Er and Deng proposed dynamic Q-Learning for on-line tuning the fuzzy inference systems. Kaya and Alhajj [29] proposed a novel multiagent reinforcement learning approach based on fuzzy OLAP association rules mining. However, these approaches encountered one or more of the following major problems: 1) the initial values of the populations were generated randomly; 2) the mutational value was generated by the constant range while the mutation point is also generated randomly; 3) the population sizes always depend on the problem which is to be solved.

In this chapter, we propose a reinforcement sequential-search-based genetic algorithm (RSSGA) method for solving above-mentioned problems. Unlike the traditional reinforcement learning, we formulate a number of time steps before failure occurs as the fitness function. The new sequential-search-based genetic algorithm (SSGA) is also proposed to perform parameter learning. Moreover, the SSGA method is different from traditional GA, which the better chromosomes will be initially generated while the better mutation points will be determined for performing efficient mutation. Compared with traditional genetic algorithm, the SSGA method generates initialize population efficiently and decides efficient mutation points to perform mutation. The advantages of the proposed R-SSGA method are summarized as follows: (1) The R-SSGA method can reduce the population sizes to a minimum size (4); (2) The chromosome which has the best performance will be chosen to perform the mutation operator in each generation. (3) The R-SSGA method converges more quickly than existing traditional genetic methods.

This chapter is organized as follows. Section 2 introduces the sequential-search-based genetic algorithm. A reinforcement sequential-search-based genetic algorithm is presented in Section 3. In Section 4, the proposed R-SSGA method is evaluated using two different control problems, and its performances are benchmarked against other structures. Finally, conclusions on the proposed algorithm are summarized in the last section.

\section{The sequential-search-based genetic algorithm}

A new genetic learning algorithm, called sequential-search-based genetic algorithm (SSGA), is proposed to adjust the parameters for the desired outputs. The proposed SSGA method is different from a traditional genetic algorithm [9], [19]. The SSGA method generates initial 
population efficiently and decides efficient mutation points to perform mutation. Like traditional genetic algorithm [9], [19], the proposed SSGA method consists of two major operators: reproduction, crossover. Before the details of these two operators are explained, coding, initialization and efficient mutation are discussed as follows:

Coding step: The first step in the SSGA method is to code a neuro-fuzzy controller into a chromosome. We adopt a Takagi-Sugeno-Kang (TSK) type neuro-fuzzy controller [13] to be the structure of the proposed SSGA method. A TSK-type neuro-fuzzy controller employs different implication and aggregation methods than the standard Mamdani controller [1]. Instead of using fuzzy sets the conclusion part of a rule, is a linear combination of the crisp inputs.

$$
\begin{gathered}
\text { IF } x_{1} \text { is } A_{1 j}\left(m_{1 j}, \sigma_{1 j}\right) \text { and } x_{2} \text { is } A_{2 j}\left(m_{2 j}, \sigma_{2 j}\right) \ldots \text { and } x_{n} \text { is } A_{n j}\left(m_{n j}, \sigma_{n j}\right) \\
\text { THEN } y^{\prime}=w_{0}+w_{1} x_{1}+\ldots+w_{i} x_{i}
\end{gathered}
$$

where $m_{i j}$ and $\sigma_{i j}$ represent a Gaussian membership function with mean and deviation with $i$ th dimension and $j$ th rule node. A fuzzy rule in Fig. 1 is represented the form in Eq. (1).

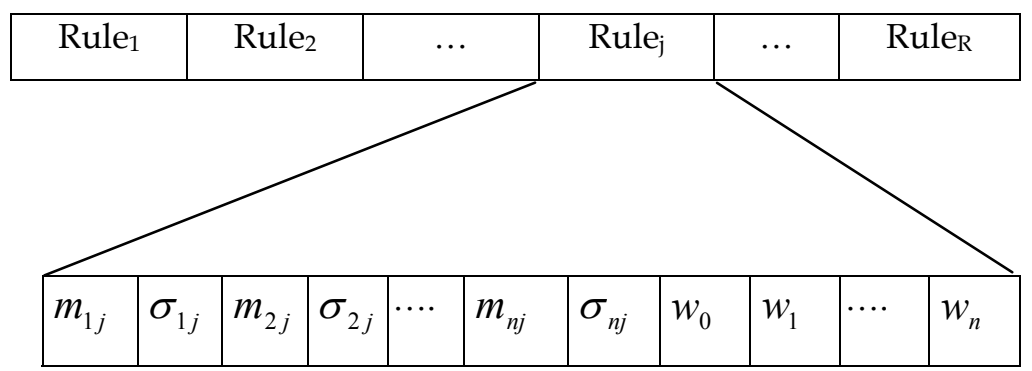

Fig. 1. Coding a fuzzy controller into a chromosome in the SSGA method.

Initialization step: Before the SSGA method is designed, individuals forming an initial population should be generated. Unlike traditional genetic algorithm, an initial population is generated randomly within a fixed range. In the SSGA method, the initial population is generated efficiently to ensure that chromosomes with good genes can be generated. The detailed steps of the initialization method are described as follows:

- Step 0: The first chromosome that represents a TSK-type fuzzy controller will be generated initially. The following formulations show how to generate the chromosomes:

$$
\begin{gathered}
\text { Deviation: } \operatorname{Ch}_{j}[p]=\operatorname{random}\left[\sigma_{\min }, \sigma_{\max }\right] \\
\text { where } \mathrm{p}=2,4,6, \ldots, 2^{*} \mathrm{n} \\
\text { Mean: } \operatorname{Ch} r_{j}[p]=\operatorname{random}\left[m_{\min }, m_{\max }\right] \\
\text { where } \mathrm{p}=1,3,5, \ldots, 2^{*} \mathrm{n}-1 \\
\text { Weight: } \mathrm{Ch} r_{j}[p]=\text { random }\left[w_{\min }, w_{\max }\right]
\end{gathered}
$$


where $p=2 * n+1, \ldots, 2 * n+(1+n)$

where $C h r_{j}$ means chromosome in $i$ th rule and $p$ represent the $p$ th gene in a $C h r_{j}$; $\left[\sigma_{\min }, \sigma_{\max }\right],\left[m_{\min }, m_{\max }\right]$, and $\left[w_{\min }, w_{\max }\right]$ represent the predefined ranges of deviation, mean, and weight. The ranges are determined by practical experimentation or trial-and-error tests.

- Step 1: To generate the other chromosomes, we use the SSGA method to generate the new chromosomes. The search algorithm of the SSGA method is similar to the local search procedure in [14]. In the SSGA method, every gene in the previous chromosomes is selected using a sequential search and the gene's value is updated to evaluate the performance based on the fitness value. The details of the SSGA method are as follows:

(a) Sequentially search for a gene in the previous chromosome.

(b) Update the chosen gene in (a) according to the following formula:

$$
\begin{aligned}
& C h r_{j}[p]=\left\{\begin{array}{l}
\operatorname{Chr}_{\mathrm{j}}[p]+\Delta\left(\text { fitness_value, } \sigma_{\max }-\mathrm{Chr}_{\mathrm{j}}[p]\right), \text { if } \alpha>0.5 \\
\mathrm{Chr}_{\mathrm{j}}[p]-\Delta\left(\text { fitness_value, } \mathrm{Chr}_{\mathrm{j}}[p]-\sigma_{\min }\right), \text { if } \alpha<0.5
\end{array}\right. \\
& \text { where } \mathrm{p}=2,4,6, \ldots, 2 * \mathrm{n} \\
& C h r_{j}[p]=\left\{\begin{array}{l}
\operatorname{Chr}_{\mathrm{j}}[p]+\Delta\left(\text { fitness_value, } m_{\max }-\mathrm{Chr}_{\mathrm{j}}[p]\right), \text { if } \alpha>0.5 \\
\mathrm{Chr}_{\mathrm{j}}[p]-\Delta\left(\text { fitness_value, } \mathrm{Chr}_{\mathrm{j}}[p]-m_{\min }\right), \text { if } \alpha<0.5
\end{array}\right. \\
& \text { where } p=1,3,5, \ldots, 2^{*} n-1 \\
& \mathrm{Chr}_{\mathrm{j}}[\mathrm{p}]=\left\{\begin{array}{l}
\mathrm{Chr}_{\mathrm{j}}[p]+\Delta\left(\text { fitness_value, } w_{\max }-\mathrm{Chr}_{\mathrm{j}}[p]\right), \text { if } \alpha>0.5 \\
\mathrm{Chr}_{\mathrm{j}}[p]-\Delta\left(\text { fitness_value, } \mathrm{Chr}_{\mathrm{j}}[p]-w_{\min }\right), \text { if } \alpha<0.5
\end{array}\right. \\
& \text { where } p=2^{*} n+1, \ldots, 2^{*} n+(1+n)
\end{aligned}
$$

where $\alpha, \lambda \in[0,1]$ are the random values; fitness_value is the fitness computed using Eq (11); $p$ represents the $p$ th gene in a chromosome; $j$ represents the $j$ th rule, respectively. The function $\Delta($ fitness _ value, $v)$ returns a value, such that $\Delta($ fitness_value, $v)$ comes close to 0 as fitness_value increases. This property causes the mutation operator to search the space uniformly during the initial stage (when fitness_value is small) and locally during the later stages, thus increasing the probability of generating children closer to its successor than a random choice and reducing the number of generations.

(c) If the new gene that is generated from (b) can improve the fitness value, then replace the old gene with the new gene in the chromosome. If not, recover the old gene in the chromosome. After this, go to (a) until every gene is selected. The pseudo code for the SSGA method is listed in Figure 2. The $C h r_{k, j}$ represents the $k$ th chromosome and 
$j$ th rule in a fuzzy controller. And $N f$ denote the size of the population, fitness $\left(C h r_{k, j n \text { new }}\right)$ is a fitness function by Eq.(11) using the $k$ th new chromosome.

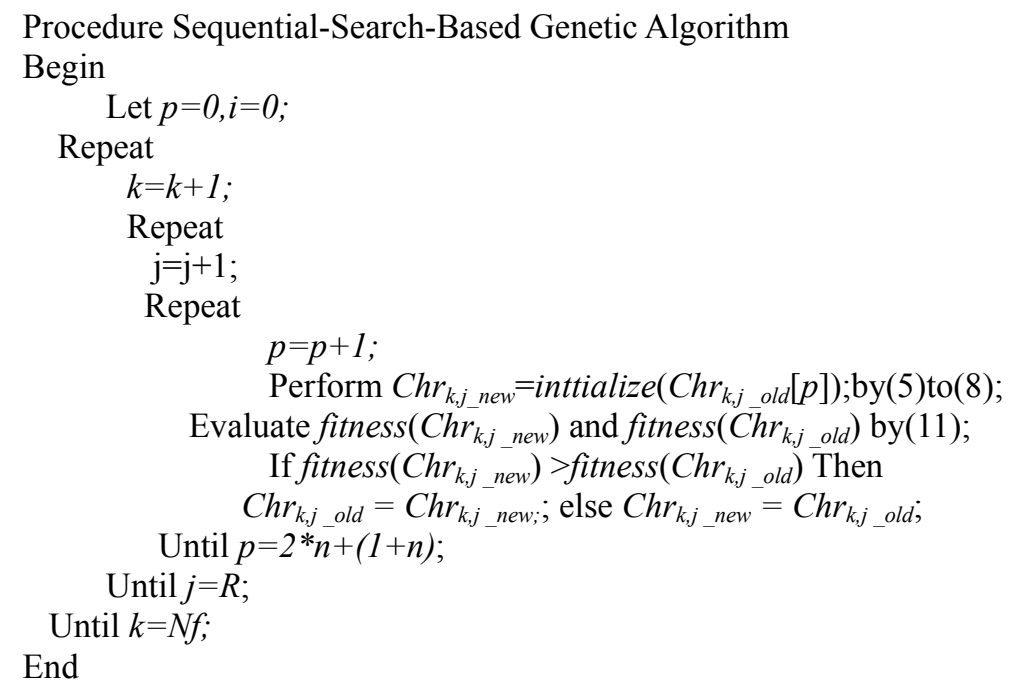

Fig. 2. The pseudo code for the SSGA method.

- Step 2: If no genes are selected to improve the fitness value in step 1, than the new chromosome will be generated according to step 0. After the new chromosome is generated, the initialization method returns to step 1 until the total number of chromosomes is generated.

The firing strength of a fuzzy rule is calculated by performing the "AND" operation on the truth values of each variable to its corresponding fuzzy sets,

$$
u_{j}=\prod_{i=1}^{n} \exp \left(-\frac{\left[u_{i}^{(1)}-m_{i j}\right]^{2}}{\sigma_{i j}^{2}}\right)
$$

where $m_{i j}$ and $\sigma_{i j}$ are, respectively, the center and the width of the Gaussian membership function of the $j$ th term of the $i$ th input variable $x_{i}$. The output of a fuzzy system is computed by 


$$
u_{\text {out }}=\frac{\sum_{j} u_{j} \sum_{i=0}^{n} w_{i j} x_{i}}{\sum_{j} u_{j}}
$$

where the weight $w_{j}$ is the output action strength associated with the $j$ th rule and $u_{\text {out }}$ is the output of the network.

Efficient mutation step: Although reproduction and crossover will produce many new strings, they do not introduce any new information to the population at the site of an individual. Mutation is an operator that randomly alters the allele of a gene. We use an efficient mutation operation, which is unlike the traditional mutation, to mutate the chromosomes. In the SSGA method, we perform efficient mutation using the best fitness value chromosome of every generation. And we use the SSGA method to decide on the mutation points. When the mutation points are selected, we use Eqs. (5) to (7) to update the genes. The efficient mutation of an individual is shown in Fig. 3.

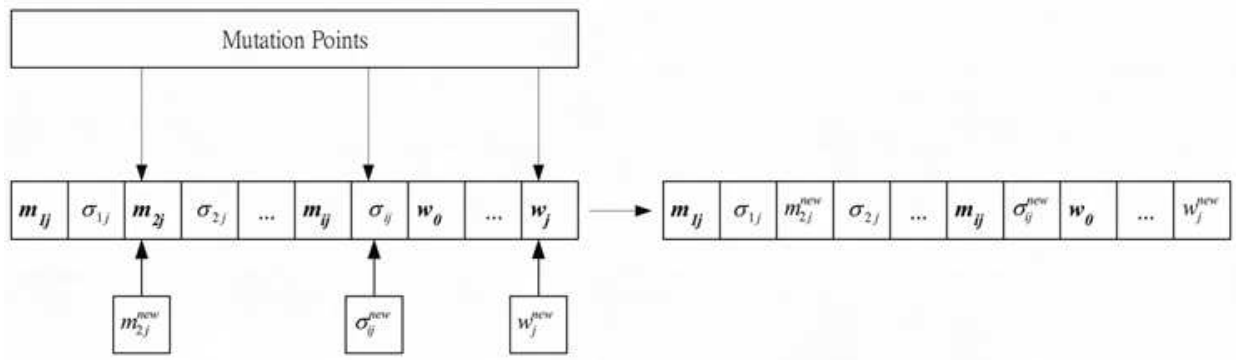

Fig. 3. Efficient mutation operation using 3 mutation points with $j$ th rule.

Reproduction step: Reproduction is a process in which individual strings are copied according to their fitness value. In this study, we use the roulette-wheel selection method [15] - a simulated roulette is spun - for this reproduction process. The best performing individuals in the top half of the population [19] advances to the next generation. The other half is generated to perform crossover and mutation operations on individuals in the top half of the parent generation.

Crossover step: Reproduction directs the search toward the best existing individuals but does not create any new individuals. In nature, an offspring has two parents and inherits genes from both. The main operator working on the parents is the crossover operator, the operation of which occurred for a selected pair with a crossover rate that was set to 0.5 in this study. The first step is to select the individuals from the population for the crossover. Tournament selection [15] is used to select the top-half of the best performing individuals [19]. The individuals are crossed and separated using a two-point crossover that is the new individuals are created by exchanging the site's values between the selected sites of parents' individual. After this operation, the individuals with poor performances are replaced by the newly produced offspring.

The aforementioned steps are done repeatedly and stopped when the predetermined condition is achieved. 


\section{Reinforcement sequential-search-based genetic algorithm (R-SSGA)}

Unlike the supervised learning problem, in which the correct "target" output values are given for each input pattern to perform neuron-fuzzy controller learning, the reinforcement learning problem has only very simple "evaluative" or "critical" information, rather than "instructive" information, available for learning. In the extreme case, there is only a single bit of information to indicate whether the output is right or wrong. Figure 4 shows how the R-SSGA method and its training environment interact in a reinforcement learning problem. The environment supplies a time-varying input vector to the R-SSGA method, receives its time-varying output/action vectors and then provides a reinforcement signal. Therefore, the reinforcement signal indicates whether a success or a failure occurs.

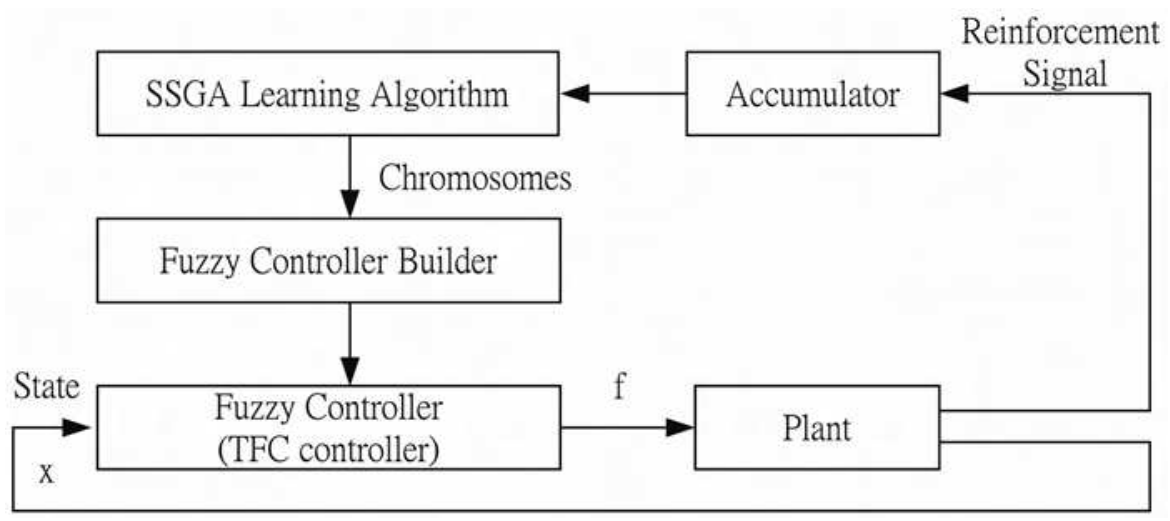

Fig. 4. The proposed R-SSGA method.

As show in Fig. 4, the R-SSGA method consists of a TSK-type fuzzy controller which acts as the control network to determine a proper action according to the current input vector (environment state). The structure of the R-SSGA method is different from Barto and his colleagues' actor-critic architecture [16]-[17]. Two neuron-like adaptive elements are integrated in this system [16]-[17]. They are the associative search element (ASE) used as a controller, and the adaptive critic element (ACE) used as a predictor. Temporal difference techniques and single-parameter stochastic exploration are used in [16]. The input to the RSSGA method is the state of the plant, and the output is a control action of the state, denoted by $f$. The only available feedback is a reinforcement signal that notifies the R-SSGA method only when a failure occurs. An accumulator plays a role which is a relative performance measure shown in Fig. 4. It accumulates the number of time steps before a failure occurs [30]. Thus, the feedback takes the form of an accumulator that determines how long the experiment is still a "success"; this is used as a relative measure of the fitness of the proposed R-SSGA method. That is, the accumulator will indicate the "fitness" of the current R-SSGA method. The key to this learning algorithm is formulating a number of time steps before failure occurs and using this formulation as the fitness function of the R-SSGA method. The advantage of the proposed method need not use the critical network as either a multi-step or single-step predictor. 


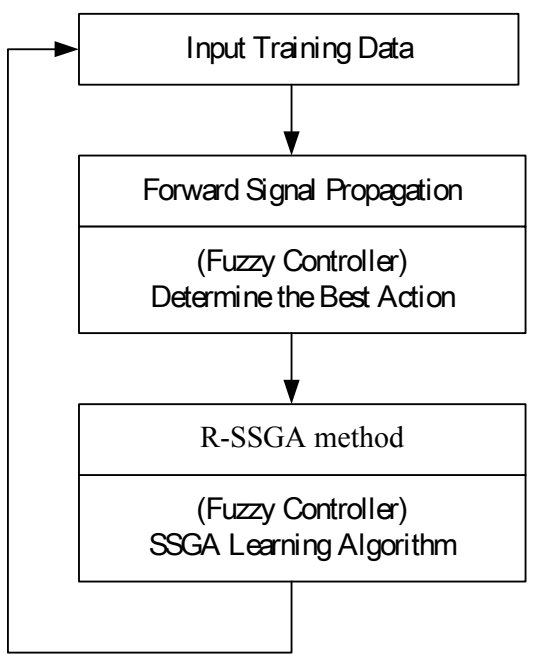

Fig. 5. Flowchart of the R-SSGA method

Figure 5 shows the flowchart of the R-SSGA method. The R-SSGA method runs in a feedforward fashion to control the environment (plant) until a failure occurs. Our relative measure of fitness function takes the form of an accumulator that determines how long the experiment is a "success". In this way, according to a defined fitness function, a fitness value is assigned to each string in the population where high fitness values means good fit. Thus, we use a number of time steps before failure occurs to define the fitness function. The fitness function is defined by:

$$
\text { Fitness_Value (i) =TIME-STEP(i) }
$$

where TIME-STEP(i) represents how long the experiment is still a "success" about the $i$ th population. Eq.(11) reflects the fact that long-time steps before failure occurs ( to keep the desired control goal longer) mean higher fitness of the R-SSGA method.

\section{Illustrative examples}

To verify the performance of the proposed R-SSGA method, two control examples - the cartpole balancing system and a water bath temperature control system-are presented in this section. For the two computer simulations, the initial parameters are given in Table 1 before training.

In this example, we shall apply the R-SSGA method to the classic control problem of the cart-pole balancing. This problem is often used as an example of inherently unstable and dynamic systems to demonstrate both modern and classic control techniques [22]-[23] or reinforcement learning schemes [18]-[19], and is now used as a control benchmark. As shown in Fig. 6, the cart-pole balancing problem is the problem of learning how to balance an upright pole. The bottom of the pole is hinged to the left or right of a cart that travels along a finite-length track. Both the cart and the pole can move only in the vertical plane; that is, each has only one degree of freedom. 
Table 1: The initial parameters before training

\begin{tabular}{ll}
\hline Parameters & Value \\
Population Size & 4 \\
Crossover Rate & 0.5
\end{tabular}

Coding Type

Real Number

$[0,1]$

$\left[\sigma_{\min }, \sigma_{\max }\right]$

$[0,1]$

$\left[m_{\min }, m_{\max }\right]$

$[-20,20]$

$\left[w_{\min }, w_{\max }\right]$

Example 1. Cart-Pole Balancing System

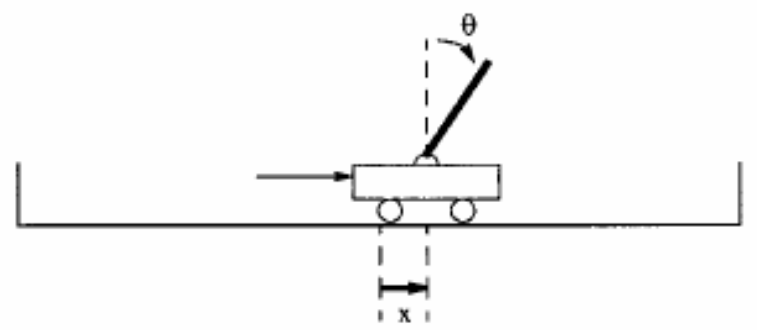

Fig. 6. The cart-pole balancing system.

There are four state variables in the system: $\theta$, the angle of the pole in an upright position

(in degrees); $\dot{\theta}$, the angular velocity of the pole (in degrees/seconds); $x$, the horizontal position of the cart's center (in meters); and $\dot{x}$, the velocity of the cart (in meters/seconds). The only control action is $f$, which is the amount of force (in Newtons) applied to the cart to move it left or right. The system fails when the pole falls past a certain angle $\left( \pm 12^{\circ}\right.$ is used here) or when the cart runs into the boundary of the track (the distance is $2.4 \mathrm{~m}$ from the center to each boundary of the track). The goal of this control problem is to determine a sequence of forces that, when applied to the cart, balance the pole so that it is upright. The motion equations that we used were: 


$$
\begin{gathered}
\theta(t+1)=\theta(t)+\Delta \dot{\theta}(t) \\
\dot{\theta}(t+1)=\dot{\theta}(t)+\Delta \frac{\left(m+m_{p}\right) g \sin \theta(t)}{(4 / 3)\left(m+m_{p}\right) l-m_{p} l \cos ^{2} \theta(t)} \\
-\frac{\cos \theta(t)\left[f(t)+m_{p} l \dot{\theta}(t)^{2} \sin \theta(t)-\mu_{c} \operatorname{sgn}(\dot{x}(t))\right]}{(4 / 3)\left(m+m_{p}\right) l-m_{p} l \cos ^{2} \theta(t)} \\
\frac{\mu_{p}\left(m+m_{p}\right) \dot{\theta}(t)}{m_{p} l} \\
-\frac{x(t+1)=x(t)+\Delta \dot{x}(t)}{(4 / 3)\left(m_{p}\right) l-m_{p} l \cos ^{2} \theta(t)} \\
x(t+1)=\dot{x}(t)+\Delta \frac{f(t)+m_{p} l\left[\dot{\theta}(t)^{2} \sin \theta(t)-\ddot{\theta}(t) \cos \theta(t)\right]}{\left(m+m_{p}\right)} \\
-\frac{\mu_{c} \operatorname{sgn}(\dot{x}(t))}{\left(m+m_{p}\right)}
\end{gathered}
$$

where

$$
l=0.5 \mathrm{~m} \text {, the length of the pole; }
$$

$m=1.1 \mathrm{~kg}$, combined mass of the pole and the cart;

$$
m_{p}=0.1 \mathrm{~kg} \text {, mass of the pole; }
$$

$g=9.8 \mathrm{~m} / \mathrm{s}$, acceleration due to the gravity;

$\mu_{c}=0.0005$, coefficient of friction of the cart on the track,

$\mu_{p}=0.000002$, coefficient of friction of the pole on the cart,

$$
\Delta=0.02(\mathrm{~s}) \text {, sampling interval. }
$$

The constraints on the variables were $-12^{\circ} \leq \theta \leq 12^{\circ},-2.4 m \leq x \leq 2.4 m$, and $10 N \leq f \leq 10 N$. A control strategy was deemed successful if it balanced a pole for 100,000 time steps.

The four input variables $(\theta, \dot{\theta}, x, \dot{x})$ and the output $f_{t}$ are normalized between 0 and 1 over 
the following ranges, $\theta \in[-12,12], \dot{\theta} \in[-60,60], x \in[-2.4,2.4], \dot{x} \in[-3,3], f_{t} \in[-10,10]$. The fitness function in this example is defined in Eq.(11) to train the R-SSGA method where Eq.(11) is used to calculate how long it takes the cart-pole balancing system to fail and receives a penalty signal of -1 when the pole falls past a certain angle $\left(|\theta|>12^{\circ} \mathrm{C}\right)$ and when the cart runs into the boundaries of the tracks falls $(|x|>2.4 m)$. In this experiment, the initial values were set to $(0,0,0,0)$. And we set four rules constitute a TSK-Type fuzzy controller.

A total of five runs were performs. Each run started at same initial state. The simulation result in Fig.7 (a) shows that the R-SSGA method learned on average to balance the pole at the 16th generation. In this figure, each run indicates that the largest fitness value in the current generation was selected before the cart-pole balancing system failed. When the proposed R-SSGA learning method is stopped, we choose the best string in the population in the final generation and tested it on the cart-pole balancing system. The final fuzzy rules generated by the R-SSGA method are described as follows:

Rule 1: If $x_{1}$ is $A_{11}(0.38,0.35)$ and $x_{2}$ is $A_{21}(5.67,0.32)$ and $x_{3}$ is $A_{31}(0.19,1.91)$ and $x_{4}$ is $A_{41}(0.40,0.825)$

THEN $y^{\prime}=-2.94+0.42 x_{1}-0.20 x_{2}-0.70 x_{3}+0.40 x_{4}$

Rule 2: IF $x_{1}$ is $A_{12}(0.52,1.70)$ and $x_{2}$ is $A_{22}(7.43,0.39)$ and $x_{3}$ is $A_{32}(0.37,14.9)$ and $x_{4}$ is $A_{42}(1.28,0.44)$

THEN $y^{\prime}=12.21+12.16 x_{1}-0.25 x_{2}+0.32 x_{3}+4.66 x_{4}$

Rule 3: IF $x_{1}$ is $A_{13}(0.52,6.66)$ and $x_{2}$ is $A_{23}(12.1,0.39)$ and $x_{3}$ is $A_{33}(0.37,9.64)$ and $x_{4}$ is $A_{43}(1.28,0.44)$

THEN $y^{\prime}=11.93+9.63 x_{1}-0.25 x_{2}+0.32 x_{3+} 9.64 x_{4}$

Rule 4: IF $x_{1}$ is $A_{14}(0.52,17)$ and $x_{2}$ is $A_{24}(9.29,0.39)$ and $x_{3}$ is $A_{34}(0.37,3.98)$

and $x_{4}$ is $A_{44}(1.28,0.44)$

THEN $y^{\prime}=11.93-3.98 x_{1}-0.25 x_{2}+0.32 x_{3}+10.29 x_{4}$ 


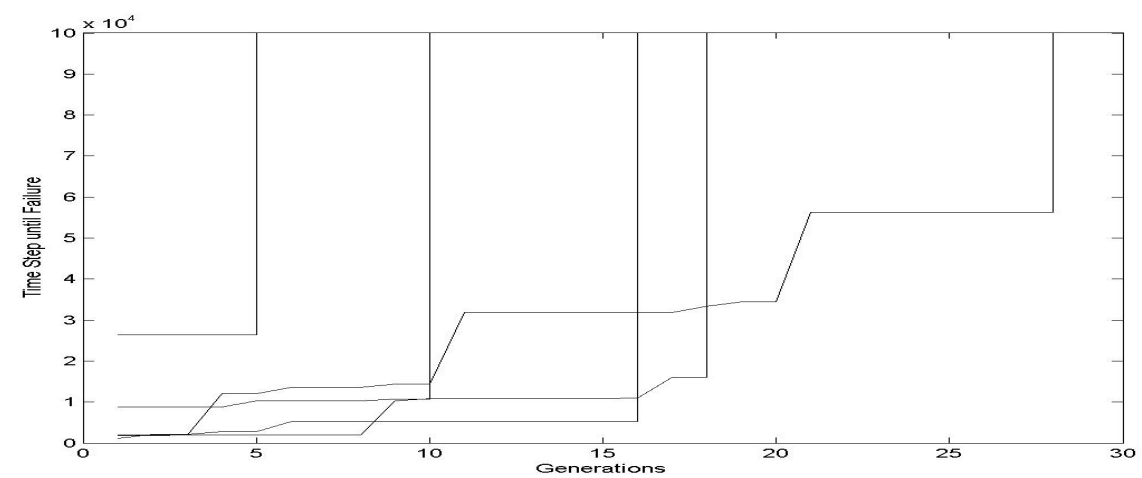

(a)

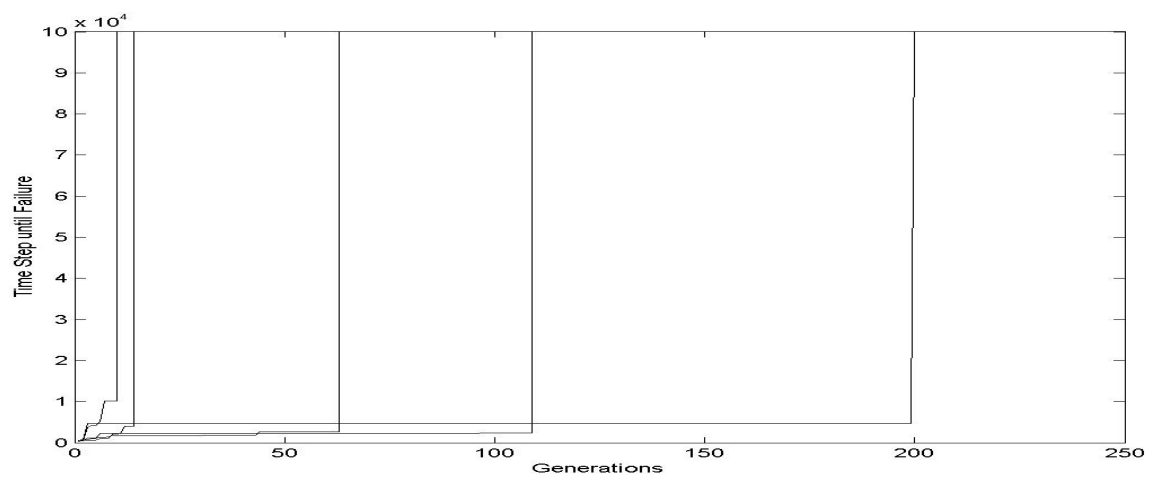

(b)

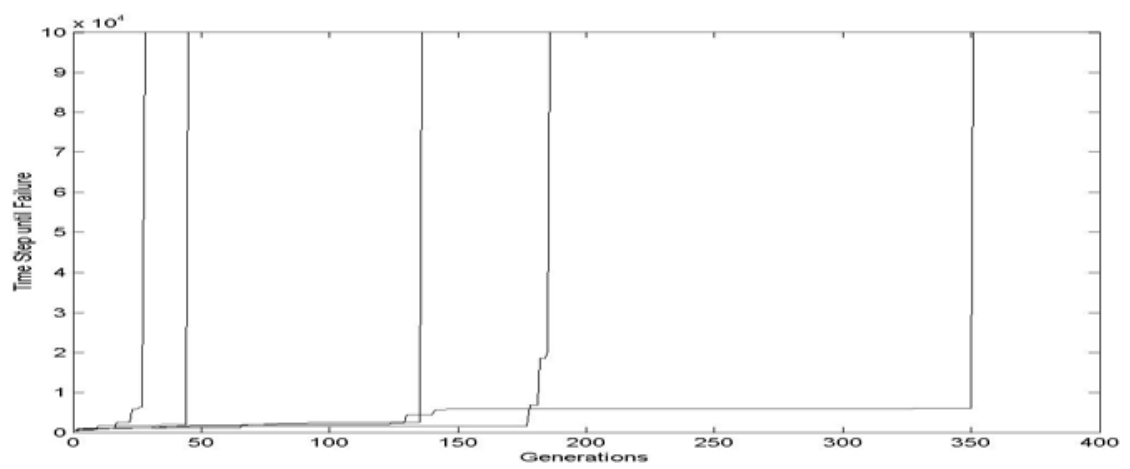

(c)

Fig. 7. The performance of (a) the R-SSGA method, (b) the SEFC method [19], and (c) the TGFC method [9] on the cart-pole balancing system.

Figure 8(a) show the angular deviation of the pole when the cart-pole balancing system was controlled by the well-trained R-SSGA method starting at the initial 
state: $r(0)=0, \dot{r}(0)=0, \theta(0)=0, \dot{\theta}(0)=0$. The average angular deviation was $0.006^{0}$.

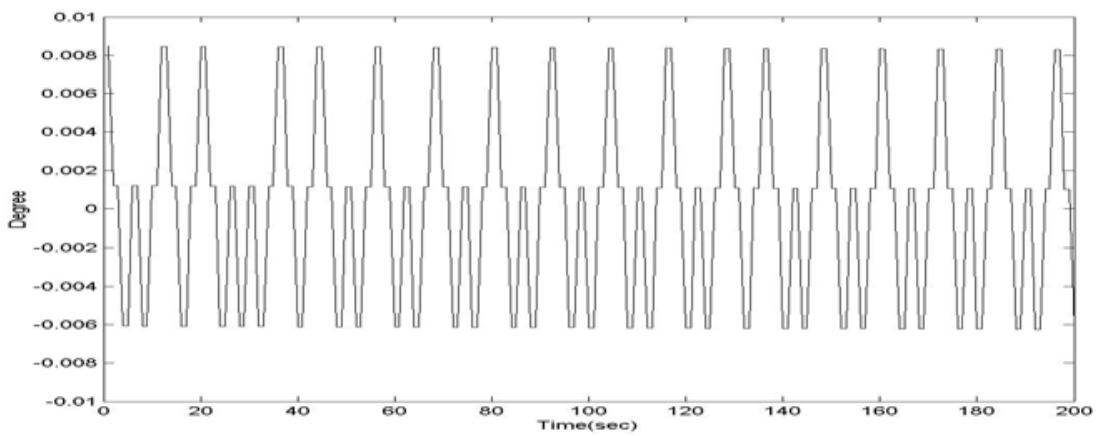

(a)

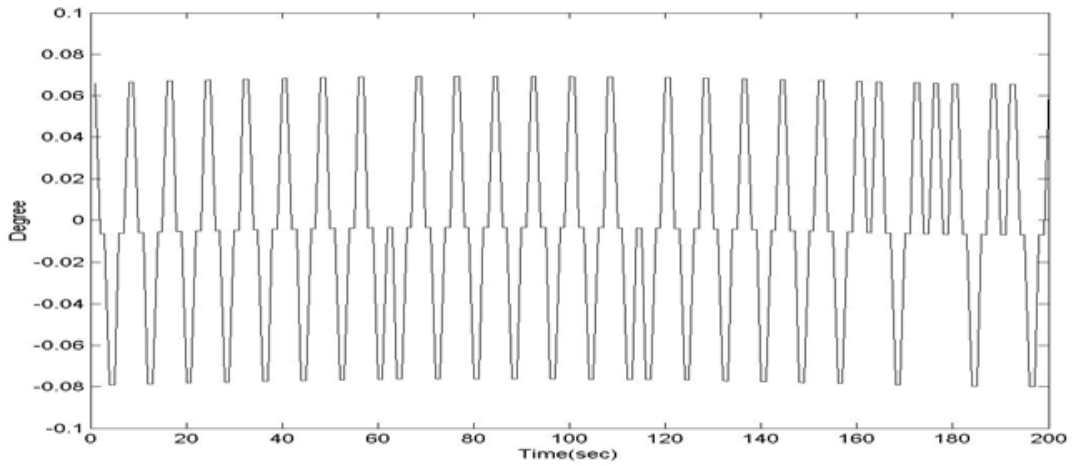

(b)

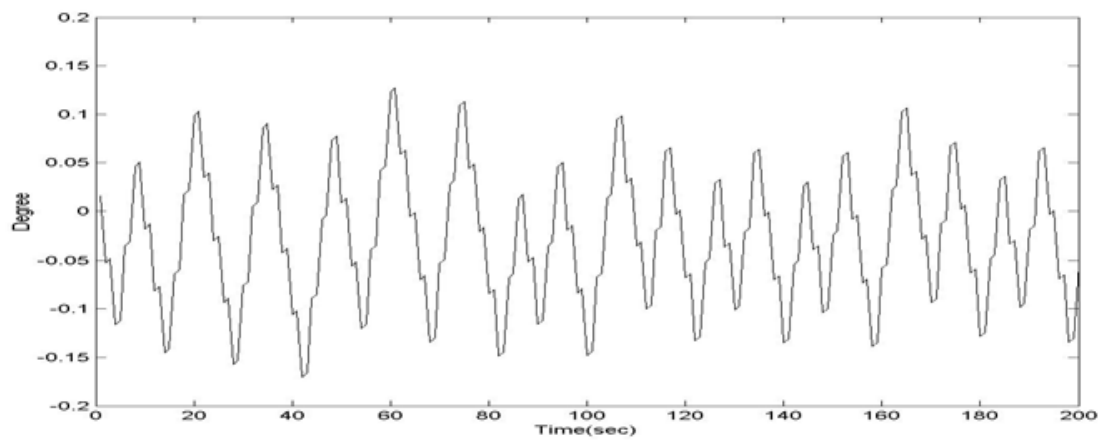

(c)

Fig. 8. Angular deviation of the pole by a trained (a) the R-SSGA method, (b) the SEFC method [19], and (c) the TGFC method [9]. 
In the experiment, we compare the performance of our system with the symbiotic evolution fuzzy controller (SEFC) [19] and the traditional genetic fuzzy controller (TGFC) [9]. In the SEFC and TGFC, the population sizes were also set to 50, and the crossover and mutation probabilities were set to 0.5 and 0.3 , respectively. Figures 7 (b) and (c) show that the SEFC method and the TGFC method learned on average to balance the pole at the $80^{\text {th }}$ and $149^{\text {th }}$ generation. In this example, we compare the CPU times of the R-SSGA method with the SEFC and the TGFC methods. Table 2 shows the CPU times of the three methods. As shown in Table 2, our method obtains shorter CPU times than the SEFC and the TGFC methods. Figures $8(\mathrm{~b})$ and 8 (c) show the angular deviation of the pole when the cart-pole balancing system was controlled by the [19] and [9] models. The average angular deviation of the [19] and [9] models were $0.06^{0}$ and $0.1^{0}$. We also try to control the cart-pole balancing system at a different initial state: $r(0)=0.6, \dot{r}(0)=0.3, \theta(0)=3, \dot{\theta}(0)=1$. Figure 9 (a)-(c) shows the angular deviation of the pole when the cart-pole balancing system was controlled by the R-SSGA, the SEFC [19], and the TGFC [9] models at the initial state: $r(0)=0.6, \dot{r}(0)=0.3, \theta(0)=3, \dot{\theta}(0)=1$.

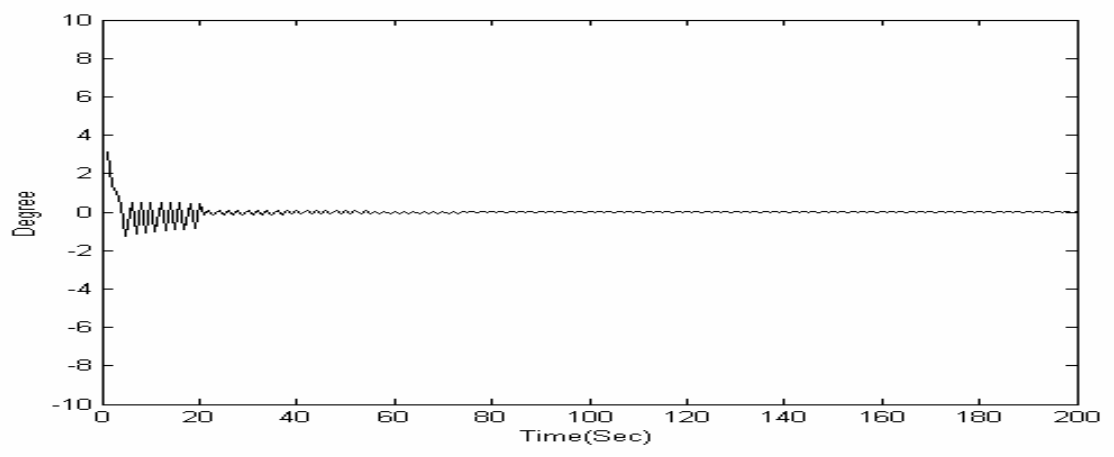

(a)

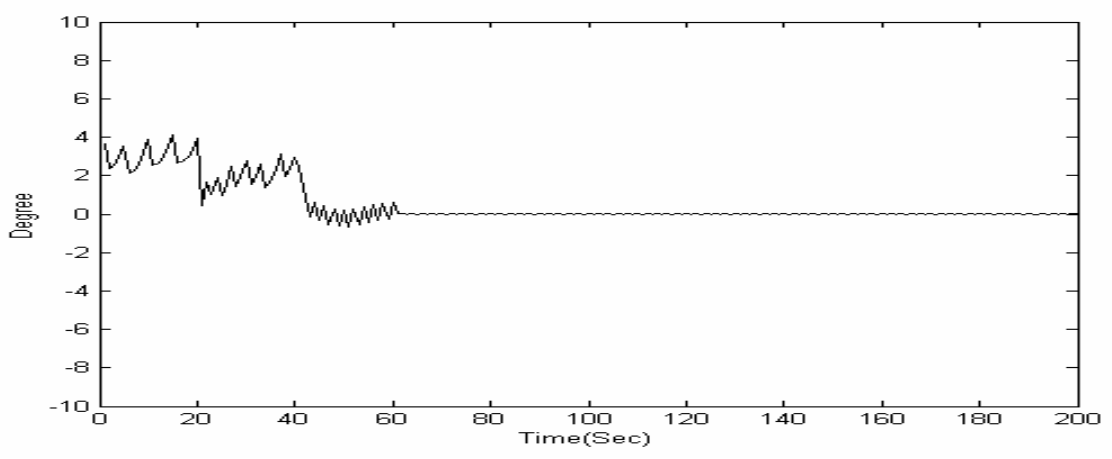

(b) 


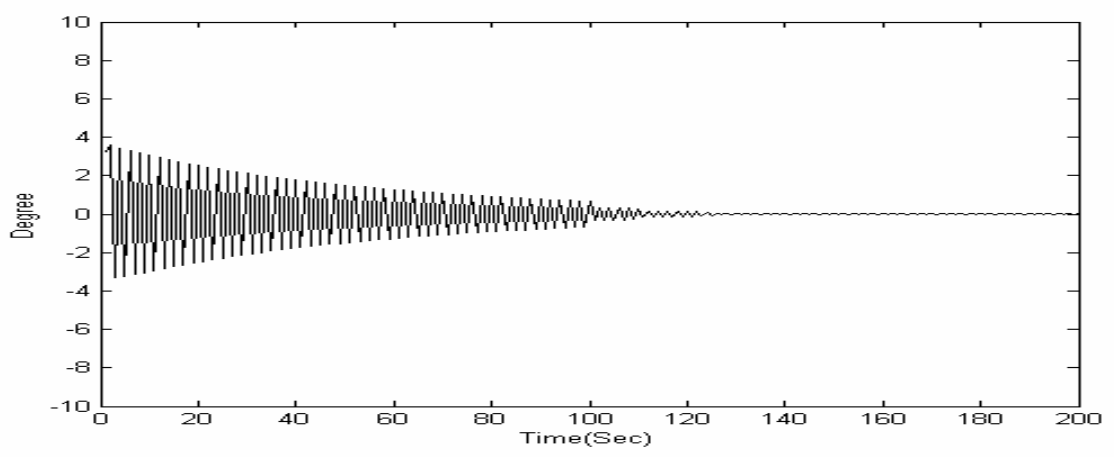

(c)

Fig. 9. Angular deviation of the pole by a trained (a) the R-SSGA method, (b) the SEFC method [19], and (c) the TGFC method [9] at the initial state: $r(0)=0.6, \dot{r}(0)=0.3, \theta(0)=3, \dot{\theta}(0)=1$.

Table 3 shows the number of pole-balance trials (which reflects the number of training episodes required) measured. The GENITOR [24] and SANE (Symbiotic Adaptive NeuroEvolution) [21] were applied to the same control problem, and the simulation results are listed in Table 3. In GENITOR, the normal evolution algorithm was used to evolve the weights in a fully connected two-layer neural network, with additional connections from each input unit to the output layer. The network has five input units, five hidden units and one output unit. In SANE, the traditional symbiotic evolution algorithm was used to evolve a two-layer neural network with five input units, eight hidden units, and two output units. An individual in SANE represents a hidden unit with five specified connections to the input and output units. In Table 3 we can see that the proposed method is feasible and effective. And the proposed R-SSGA method only took 4 rules and the population size was 4 .

\begin{tabular}{cccc}
\hline \hline Method & Mean (Sec) & Best (Sec) & Worst (Sec) \\
\hline \hline R-SSGA & $\mathbf{2 0}$ & $\mathbf{3}$ & $\mathbf{6 0}$ \\
SEFC [19] & 36 & 4 & 236 \\
TGFC [9] & 165 & 8 & 412 \\
\hline \hline
\end{tabular}

Table 2. Performance comparison of the R-SSGA, the SEFC, and the TGFC methods. 


\begin{tabular}{cccc}
\hline Method & Mean & Best & Worst \\
\hline \hline GENITOR [24] & 2578 & 415 & 12964 \\
SANE [21] & 1691 & 46 & 4461 \\
TGFC [9] & 80 & 26 & 200 \\
SEFC [19] & 149 & 10 & 350 \\
R-SSGA & $\mathbf{1 7}$ & $\mathbf{5}$ & $\mathbf{2 9}$ \\
\hline \hline
\end{tabular}

Table 3. Performance comparison of various existing models in Example 1.

In this example, to verify the performance of our proposed method, we use five different initial states for the R-SSGA, the SEFC, and the TGFC methods. The five different initial states are shown as follows:

$$
\begin{aligned}
& \text { S1: } r(0)=0.8, \dot{r}(0)=0.2, \theta(0)=8, \dot{\theta}(0)=3 \\
& \text { S2: } r(0)=0.3, \dot{r}(0)=0.1, \theta(0)=2, \dot{\theta}(0)=0 \\
& \text { S3: } r(0)=0.5, \dot{r}(0)=0.1, \theta(0)=4, \dot{\theta}(0)=2 \\
& \text { S4: } r(0)=0.7, \dot{r}(0)=0.4, \theta(0)=6, \dot{\theta}(0)=3 \\
& \text { S5: } r(0)=0.2, \dot{r}(0)=0.1, \theta(0)=2, \dot{\theta}(0)=1
\end{aligned}
$$

Figure 10 (a)-(c) show that the R-SSGA, the SEFC, and the TGFC methods learned on average to balance the pole at the $78^{\text {th }}, 105^{\text {th }}$, and $166^{\text {th }}$ generation. Figure $11(\mathrm{a})$-(c) show the angular deviation of the pole when the cart-pole balancing system was controlled by the RSSGA method, the SEFC method [19], and the TGFC method [9] that starting at the initial state: $r(0)=0, \dot{r}(0)=0, \theta(0)=0, \dot{\theta}(0)=0$. The average angular deviations were $0.01^{0}, 0.04^{\circ}$, and $0.08^{\circ}$. Table 4 shows the number of pole-balance trials measured of the RSSGA, the SEFC [19], and the TGFC [9] methods. In Table 4, we see that the proposed method obtains a better performance than some existing methods [9], [19]. 


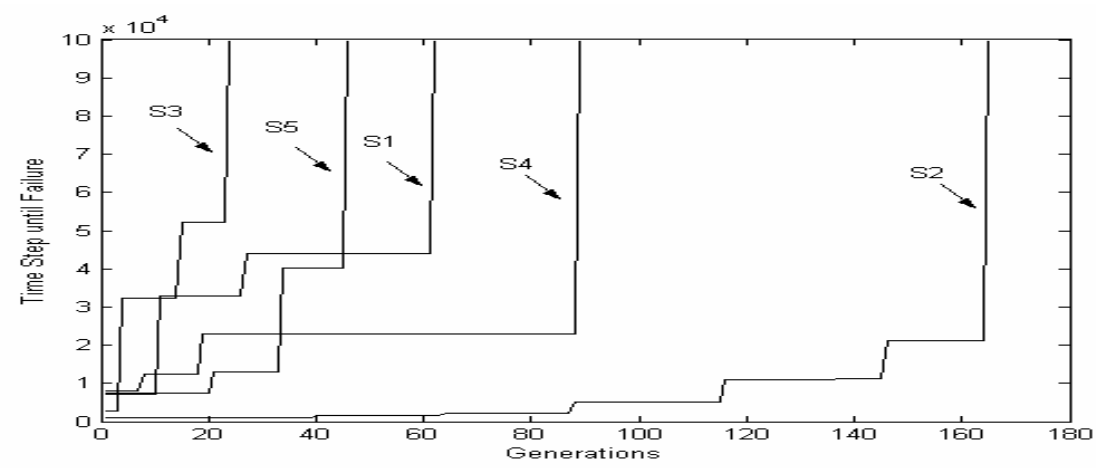

(a)

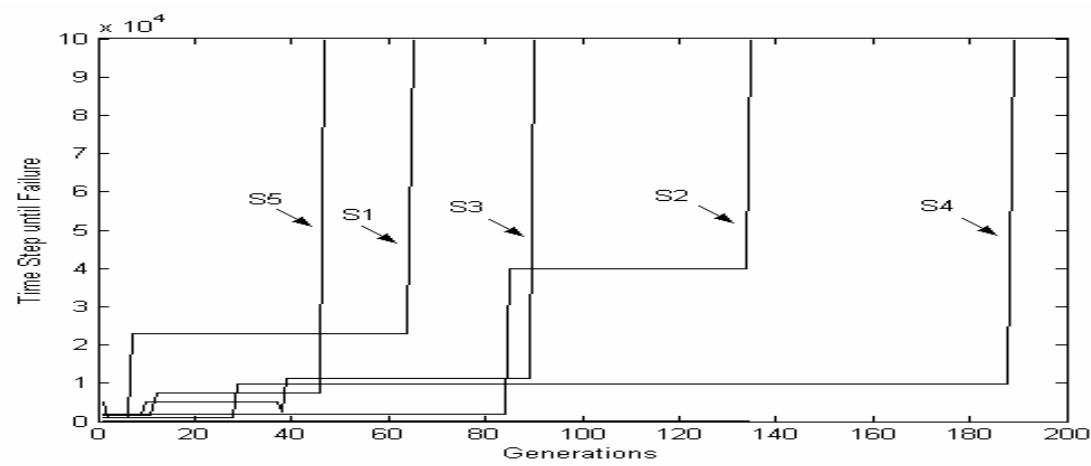

(b)

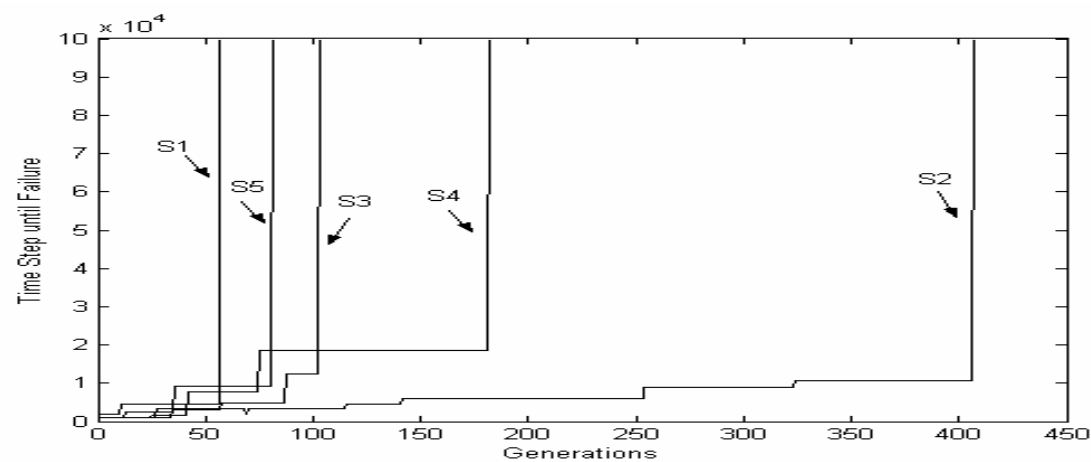

(c)

Fig. 10. The performance of (a) the R-SSGA method, (b) the SEFC method [19], and (c) the TGFC method [9] on the cart-pole balancing system starting at five different initial states. 


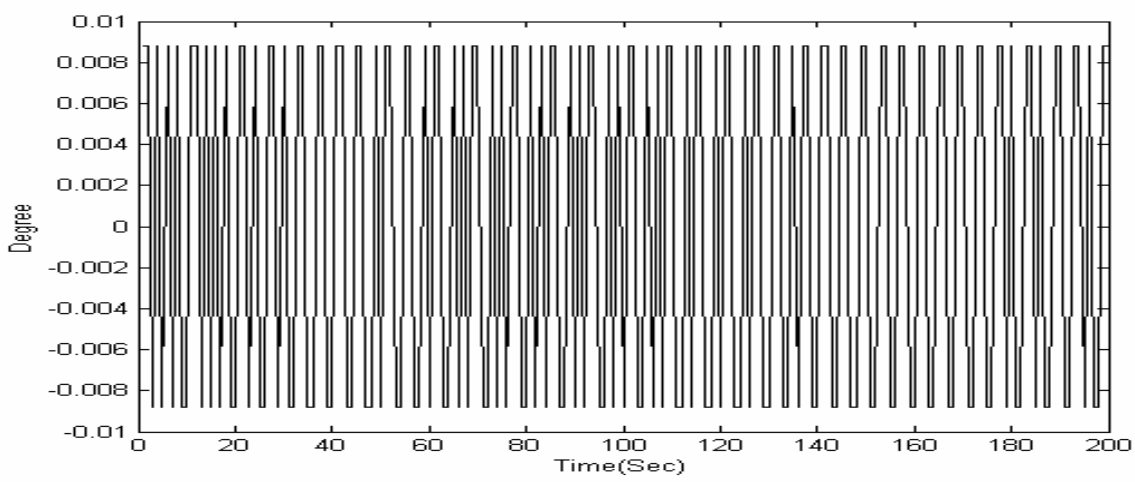

(a)

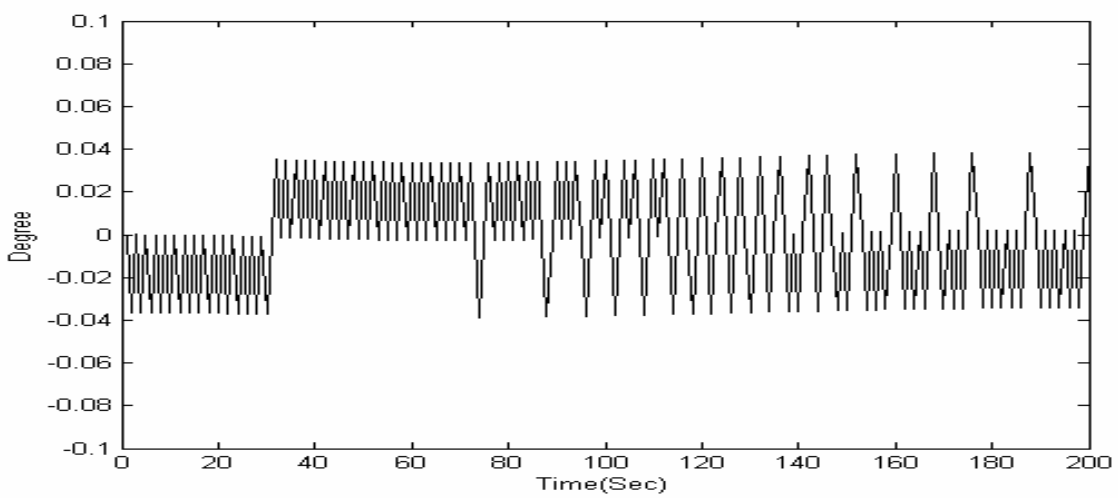

(b)

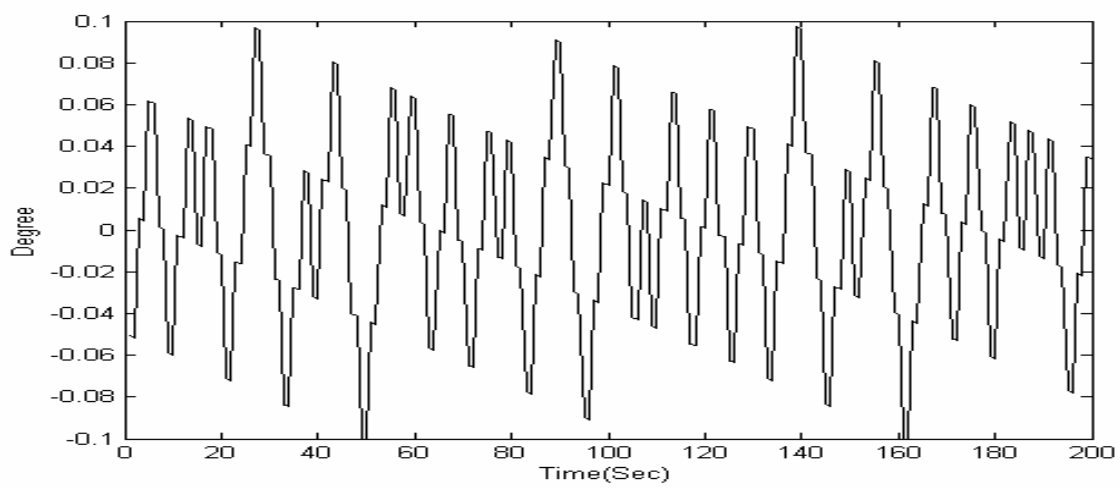

(c)

Fig. 11. Angular deviation of the pole by a trained (a) the R-SSGA method, (b) the SEFC method [19], and (c) the TGFC method [9]. 
Table 4: Performance comparison of existing models in Example 1.

\begin{tabular}{cccc}
\hline \hline Method & Mean & Best & Worst \\
TGFC [9] & 166 & 57 & 407 \\
SEFC [19] & 105 & 47 & 189 \\
R-SSGA & $\mathbf{7 8}$ & $\mathbf{2 4}$ & $\mathbf{1 6 5}$ \\
\hline
\end{tabular}

Example 2. Water Bath Temperature Control System

The goal of this simulation was to control the temperature of a water bath system given by

$$
\frac{d y(t)}{d t}=\frac{u(t)}{C}+\frac{Y_{0}-y(t)}{R C}
$$

where $y(t)$ is the system output temperature in ${ }^{\circ} C ; u(t)$ is the heat flowing into the system; $Y_{0}$ is the room temperature; $C$ is the equivalent system thermal capacity; and $R$ is the equivalent thermal resistance between the system borders and the surroundings.

Assuming that $R$ and $C$ are essentially constant, we rewrite the system in Eq.(17) into discrete-time form with some reasonable approximation. The system

$$
y(t+1)=e^{-\alpha T s} y(k)+\frac{\frac{\beta}{\alpha}\left(1-e^{-\alpha T s}\right)}{1+e^{0.5 y(k)-40}} u(k)+\left[1-e^{-\alpha T s}\right] y_{0}
$$

is obtained, where $\alpha$ and $\beta$ are constant values describing $R$ and $C$. The system parameters used in this example were $\alpha=1.0015 e^{-4}, \beta=8.67973 e^{-3}$, and $Y_{0}=25.0\left({ }^{\circ} \mathrm{C}\right.$ ), which were obtained from a real water bath plant in [3]. The input $u(k)$ was limited to 0 , and the voltage was $5 \mathrm{~V}$. The sampling period was $T_{s}=30$. The system configuration is shown in Fig. 12, where $y_{\text {ref }}$ was the desired temperature of the controlled plant.

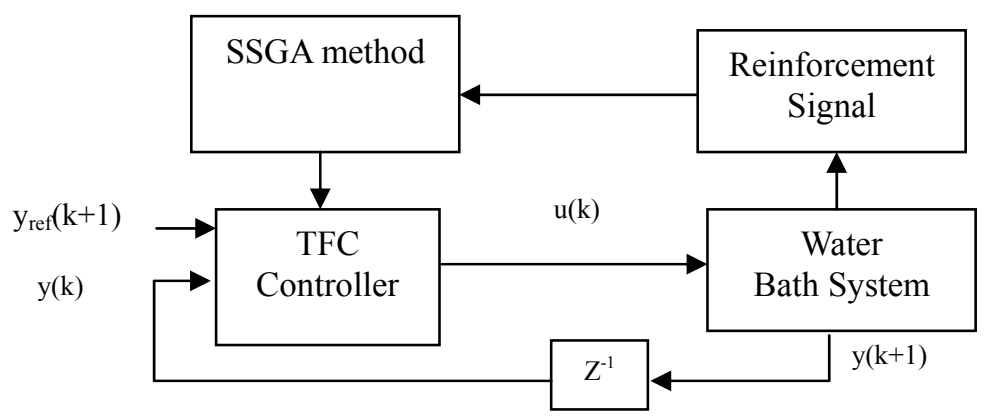

Fig. 12. Flow diagram of using the R-SSGA method for solving the temperature control problem. 
In this example, $y_{\text {ref }}$ and $y(k)$ and the output $u(k)$ were normalized between 0 and 1 over the following ranges: $y_{\text {ref }}:[25,85], y(k):[25,85]$, and $u(k):[0,5]$. The values of floating-point numbers were initially assigned using the R-SSGA method initially. The fitness function was set for each reassigned regulation temperature $T=35,55$, and 75 , starting from the current temperature and again after 10 time steps. The control temperature error should be within $\pm 1.5^{\circ} \mathrm{C}$; otherwise failure occurs. In the R-SSGA method, we set five rules constitute a TSK-Type fuzzy controller using the proposed R-SSGA method. A total of five runs were performed. Each run started at same initial state.

The simulation result in Fig. 13(a) shows that the R-SSGA method learned on average to success at the 25th generation. In this figure, each run indicates that the largest fitness value in the current generation was selected before the water bath temperature system failed. When the R-SSGA learning is stopped, we chose the best string in the population in the final generation and tested it with two different examples in the water bath temperature control system. The final fuzzy rules of a TSK-Type fuzzy controller by the R-SSGA method are described as follows:

Rule 1: IF $x_{1}$ is $A_{11}(1.23,0.75)$ and $x_{2}$ is $A_{21}(0.13,0.81)$

THEN $y^{\prime}=7.09+8.50 x_{1}+1.51 x_{2}$

Rule 2: IF $x_{1}$ is $A_{12}(0.18,0.352)$ and $x_{2}$ is $A_{22}(1.09,0.45)$

THEN $y^{\prime}=-19.41-14.051 x_{1}-16.81 x_{2}$

Rule 3: IF $x_{1}$ is $A_{13}(0.19,0.36)$ and $x_{2}$ is $A_{23}(1.10,0.46)$

THEN $y^{\prime}=-19.42-14.05 x_{1}-16.80 x_{2}$

Rule 4: IF $x_{1}$ is $A_{14}\left(0.0001\right.$ 1.27) and $x_{2}$ is $A_{24}(1.09,0.45)$

THEN $y^{\prime}=5.40+8.47 x_{1}-16.81 x_{2}$

Rule 5: IF $x_{1}$ is $A_{15}(5.0,0.66)$ and $x_{2}$ is $A_{25}(0.14,0.08)$

THEN $y^{\prime}=-4.85-5.88 x_{1}+9.45 x_{2}$

where $A_{i j}\left(m_{i j}, \sigma_{i j}\right)$ represents a Gaussian membership function with mean $m_{i j}$ and deviation $\sigma_{i j}$ in $i$ th input dimension and $j$ th rule. In this example, as with example 1 , we also compare the performance of our system with the SEFC method [19] and the TGFC method [9]. Figures 13 (b) and 10(c) show the performance of [19] and [9] methods. In this 
figure we can see that the SEFC and TGFC methods learned on average to balance the pole at the 49 th and 96 th generation but in our model just take 25 generations.

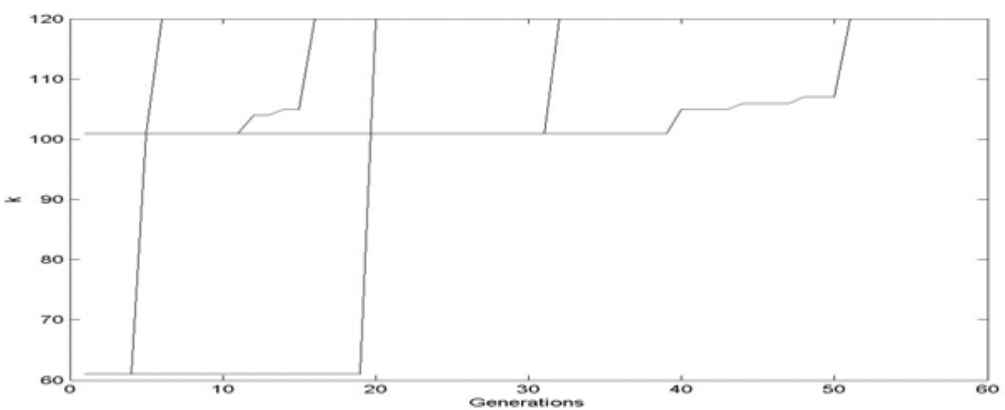

(a)

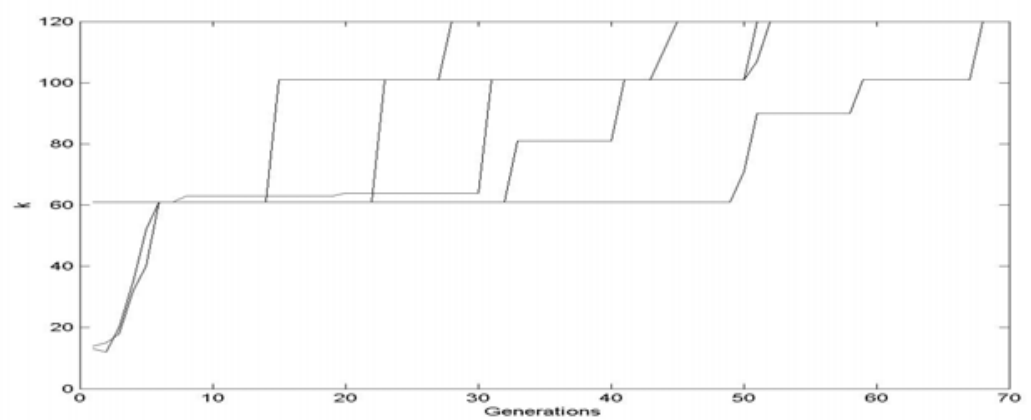

(b)

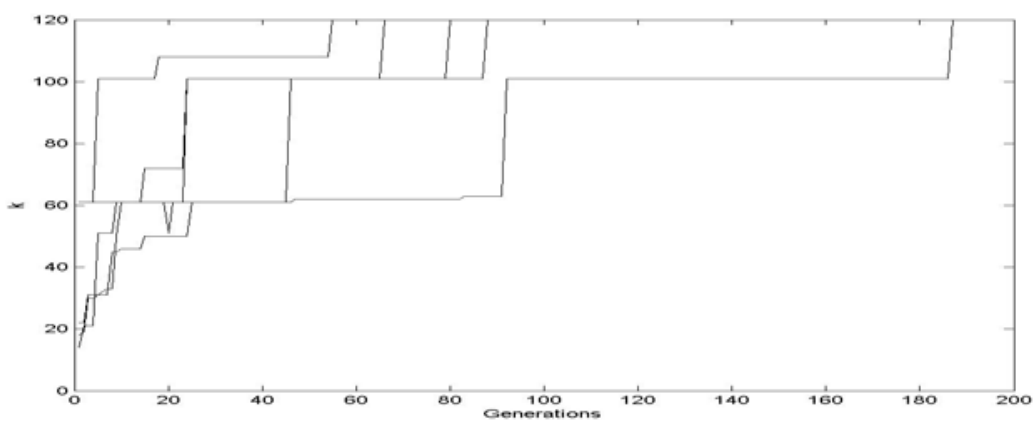

(c)

Fig. 13. The performance of the water bath system for (a) the R-SSGA method, (b) the SEFC method [19] and, (c) the TGFC method [9].

For testing the controller system, we compare the three methods (the R-SSGA, SEFC, and TGFC methods). The three methods are applied to the water bath temperature control 
system. The comparison performance measures included a set points regulation and a change of parameters.

The first task was to control the simulated system to follow three set points

$$
y_{\text {ref }}(k)=\left\{\begin{array}{llc}
35^{\circ} c, & \text { for } & k \leq 40 \\
55^{\circ} c, & \text { for } & 40<k \leq 80 \\
75^{\circ} c, & \text { for } & 80<k \leq 120 .
\end{array}\right.
$$

The regulation performance of the R-SSGA method is shown in Fig. 14(a). The error curves of the three methods are shown in Fig. 14(b). In this figure, the R-SSGA method obtains smaller errors than others.

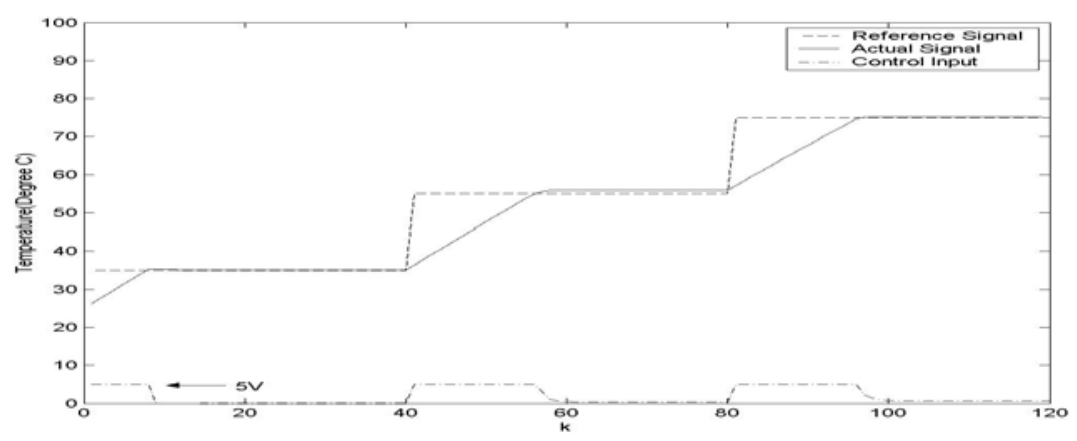

(a)

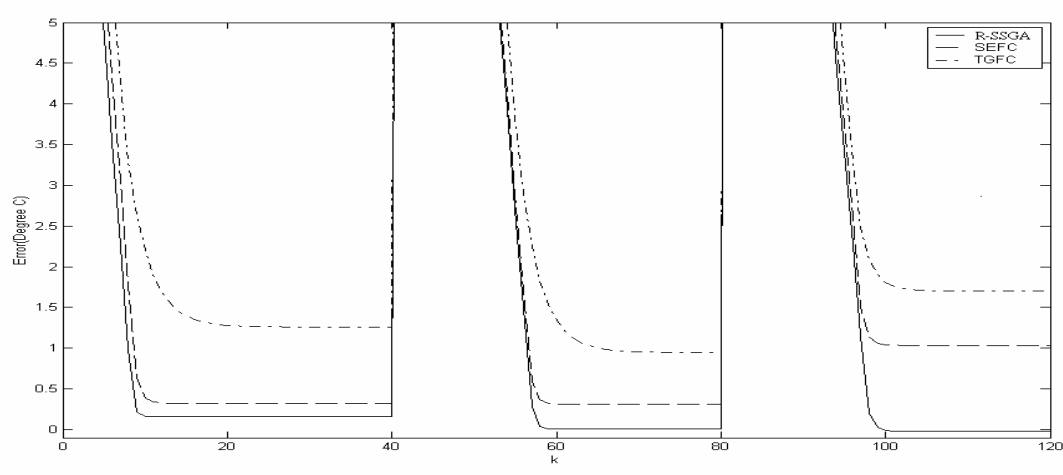

(b)

Fig. 14. (a) Final regulation performance of the R-SSGA method for water bath system. (b) The error curves of the R-SSGA method, the SEFC method and the TGFC method.

In the second set of simulations, the tracking capability of the R-SSGA method with respect to ramp-reference signals is studied. We define 


$$
y_{\text {ref }}(k)=\left\{\begin{array}{lll}
34^{\circ} \mathrm{C} & \text { for } & k \leq 30 \\
(34+0.5 *(k-30))^{\circ} \mathrm{C} & \text { for } & 30<k \leq 50 \\
(44+0.8 *(k-50))^{\circ} \mathrm{C} & \text { for } & 50<k \leq 70 \\
(60+0.5 *(k-70))^{\circ} \mathrm{C} & \text { for } & 70<k \leq 90 \\
70^{\circ} \mathrm{C} & \text { for } & 90<k \leq 120
\end{array}\right.
$$

The tracking performance of the R-SSGA method is shown in Fig. 15(a). The corresponding errors of the three methods are shown in Fig. 15(b). The results show the good control and disturbance rejection capabilities of the trained R-SSGA method in the water bath system.

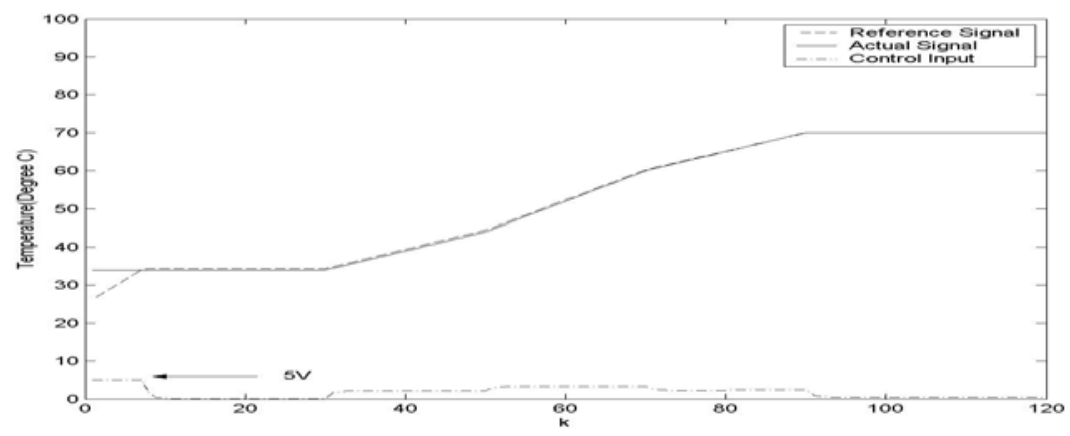

(a)

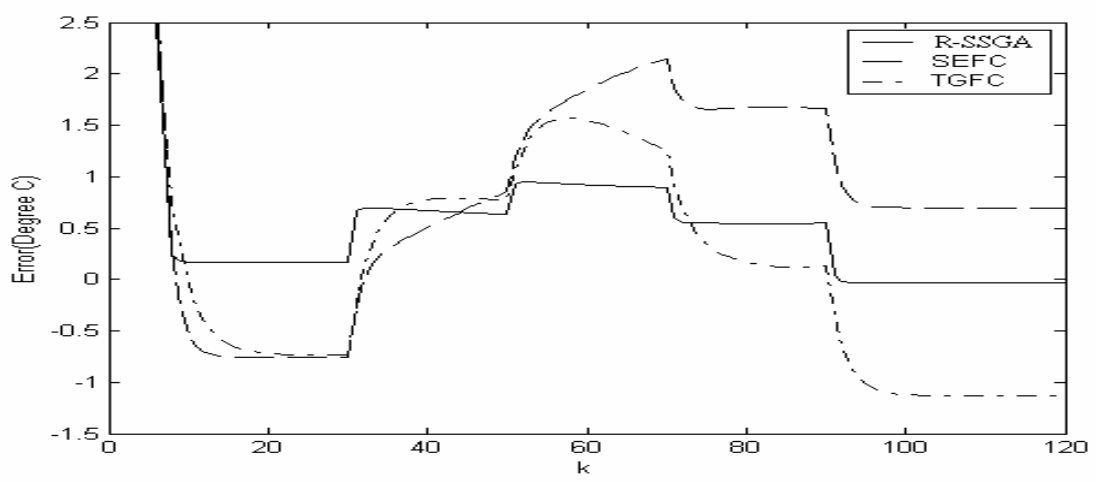

(b)

Fig. 15. (a) The tracking of the R-SSGA method when a change occurs in the water bath system. (b) The error curves of the R-SSGA method, the SEFC method [19], and the TGFC method [9].

To test their regulation performance, a performance index, sum of absolute error (SAE), is defined by 


$$
S A E=\sum_{k}\left|y_{r e f}(k)-y(k)\right|
$$

where $y_{\text {ref }}(k)$ and $y(k)$ are the reference output and the actual output of the simulated system, respectively. Table 5 shows the comparison the SAE among the R-SSGA method, the SEFC method, and the TGFC method. As show in Table 5, the proposed R-SSGA method has better performance than that of the others. And the proposed method only takes 5 rules and the populations' size is minimized to 4 .

$$
S A E=\sum_{k=1}^{120}\left|y_{r e f}(k)-y(k)\right| \quad \text { R-SSGA } \quad \text { SEFC [19] TGFC [9] }
$$

$\begin{array}{cccc}\text { Regulation Performance } & \mathbf{3 6 0 . 0 4} & 370.12 & 400.12 \\ \text { Tracking Performance } & \mathbf{5 4 . 1 8 7} & 90.81 & 104.221\end{array}$

Table 5: Performance comparison of various existing models in Example 2.

\section{Conclusions}

A novel reinforcement sequential-search-based genetic algorithm (R-SSGA) is proposed. The better chromosomes will be initially generated while the better mutation points will be determined for performing efficient mutation. We formulate a number of time steps before failure occurs as the fitness function. The proposed R-SSGA method makes the design of TSK-Type fuzzy controllers more practical for real-world applications, since it greatly lessens the quality and quantity requirements of the teaching signals. Two typical examples were presented to show the fundamental applications of the proposed R-SSGA method. Simulation results have shown that 1 ) the R-SSGA method converges quickly; 2) the R-SSGA method requires a small number of population sizes (only 4); 3) the R-SSGA method obtains a smaller average angular deviation than other methods.

\section{Acknowledgement}

This research is supported by the National Science Council of R.O.C. under grant NSC 952221-E-324- 028-MY2.

\section{References}

C. T. Lin and C. S. G. Lee, Neural Fuzzy Systems: A neural-fuzzy synergism to intelligent systems. Englewood Cliffs, NJ: Prentice-Hall, May 1996. (with disk).

C. L. Karr and E. J. Gentry, "Fuzzy control of ph using genetic algorithms," IEEE Trans. on Fuzzy Syst., vol. 1, pp. 46-53, Feb. 1993.

J. Tanomaru and S. Omatu, "Process control by on-line trained neural controllers," IEEE Trans. on Ind. Electron., Vol. 39, pp. 511-521, 1992.

K.J. ${ }^{\circ}$ Astrom and B. Wittenmark, Adaptive Control. Reading, MA: Addison-Wesley, 1989.

C. J. Lin and C. H. Chen, "Nonlinear system control using compensatory neuro-fuzzy networks," IEICE Trans. Fundamentals, vol. E86-A, no. 9, pp. 2309-2316, Sept. 2003. 
C. F. Juang and C. T. Lin, "An online self-constructing neural fuzzy inference network and its applications," IEEE Trans. Fuzzy Syst., vol. 6, no. 1, pp.12-32, Feb. 1998.

C. W. Anderson, "Learning and problem solving with multilayer connectionist systems," Ph.D. dissertation, Univ. Massachusetts, Amherst, 1986.

A. G. Barto and M. I. Jordan, "Gradient following without backpropagation in layered networks," in Proc. IEEE 1st Annual Conf. Neural Networks, vol. 2, San Diego, CA, pp. 629-636, 1987.

C. T. Lin and C. P. Jo, "GA-based fuzzy reinforcement learning for control of a magnetic bearing system," IEEE Trans. Syst., Man, Cybern., Part B, vol. 30, no. 2, pp. 276-289, Apr. 2000.

X. W. Yan, Z.D. Deng and Z.Q. Sun, "Competitive Takagi-Sugeno fuzzy reinforcement learning," in Proc. IEEE Int. Conf. Control Applications., pp. 878-883, Sept. 2001.

G.rigore O., "Reinforcement learning neural network used in control of nonlinear systems, " in Proc. IEEE Int. Conf. Industrial Technology., vol. 1, pp. 19-22, Jan. 2000.

X. Xu and H. G. He, "Residual-gradient-based neural reinforcement learning for the optimal control of an acrobat," in Proc. IEEE Int. Conf. Intelligent Control., pp. 27-30, Oct. 2002.

T. Takagi, M. Sugeno, "Fuzzy identification of systems and its applications to modeling and control", IEEE Trans. Syst., Man, Cybern., vol. 1, no. 1, pp. 116-32, 1985.

J.-S. R. Jang, C. T. Sun, and E. Mizutani, Neuro-Fuzzy and Soft Computing, Ch. 17, PrenticeHall, 1997.

O. Cordon, F. Herrera, F. Hoffmann, and L. Magdalena, Genetic fuzzy systems evolutionary tuning and learning of fuzzy knowledge bases. Advances in Fuzzy SystemsApplications and Theory, vol.19, NJ: World Scientific Publishing, 2001.

A. G. Barto and R. S. Sutton, "Landmark learning: An illustration of associative search, " Biol. Cybern. Vol. 42, pp. 1-8, 1981.

A. G. Barto, R. S. Sutton, and C. W. Anderson, "Neuron like adaptive elements that can solve difficult learning control problem," IEEE Trans. Syst., Man, Cybern., vol. SMC13, no 5, pp. 834-847, 1983.

C. T. Lin and C. S. G. Lee, "Reinforcement structure/parameter learning for neural-networkbased fuzzy logic control systems," IEEE Trans. Fuzzy Syst., vol. 2, pp. 46-63, Feb. 1994.

C. F. Juang, J. Y. Lin and C. T. Lin, "Genetic reinforcement learning through symbiotic evolution for fuzzy controller design," IEEE Trans. Syst., Man, Cybern., Part B, vol. 30, no. 2, pp. 290-302, Apr. 2000.

A. Homaifar and E. McCormick, "Simultaneous design of membership functions and rule sets for fuzzy controllers using genetic algorithms," IEEE Trans. Fuzzy Syst., vol. 3, no. 9, pp. 129-139, May 1995.

D. E. Moriarty and R. Miikkulainen, "Efficient reinforcement learning through symbiotic evolution," Mach. Learn., vol. 22, pp. 11-32, 1996.

R. H. Cannon, Jr., Dynamics of Physical Systems. New York: Mc- Graw-Hill, 1967.

K. C. Cheok and N. K. Loh, "A ball-balancing demonstration of optimal and disturbanceaccommodating control," IEEE Contr. Syst. Mag., pp. 54-57, 1987.

D. Whitley, S. Dominic, R. Das, and C. W. Anderson, "Genetic reinforcement learning for neuro control problems," Mach. Learn., vol. 13, pp. 259-284, 1993.

J. Hauser, S. Sastry, and P. Kokotovic, "Nonolinear control via approximate input-output 
linearization: the ball and beam example," IEEE Trans. Automatic Control, vol. 37, pp. 392-398, Mar. 1992.

C. F. Juang, "A TSK-type recurrent fuzzy network for dynamic systems processing by neural network and genetic algorithms," IEEE Trans. on Fuzzy Systems, Vol. 10, No. 2, pp. 155-170, April, 2002.

C. J. Lin, "A GA-based neural fuzzy system for temperature control," Fuzzy Sets and Systems, Vol. 143, pp. 311-333, 2004.

M. J. Er and C. Deng, "Online tuning of fuzzy inference systems using dynamic QLearning," IEEE Trans. Syst., Man, Cybern., Part B, vol. 34, no. 3, pp. 1478-1489, June 2004.

M. Kaya, R. Alhajj, “Fuzzy OLAP association rules mining-based modular reinforcement learning approach for multiagent systems," IEEE Trans. on Syst., Man, Cybern., Part B, vol. 35, no. 2, pp. 326-338, Apr. 2005.

C. J. Lin, "A GA-based neural network with supervised and reinforcement learning," Journal of The Chinese Institute of Electrical Engineering, Vol. 9, No. 1, pp.11-24. 


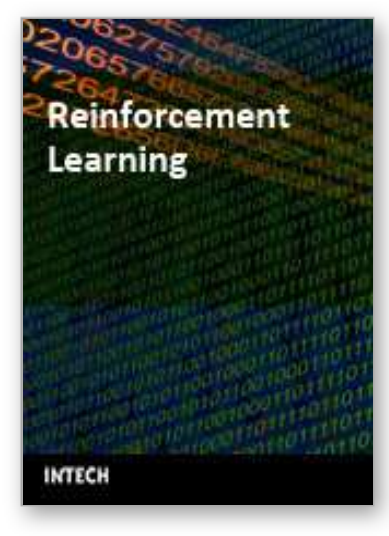

\author{
Reinforcement Learning \\ Edited by Cornelius Weber, Mark Elshaw and Norbert Michael Mayer
}

ISBN 978-3-902613-14-1

Hard cover, 424 pages

Publisher I-Tech Education and Publishing

Published online 01, January, 2008

Published in print edition January, 2008

Brains rule the world, and brain-like computation is increasingly used in computers and electronic devices. Brain-like computation is about processing and interpreting data or directly putting forward and performing actions. Learning is a very important aspect. This book is on reinforcement learning which involves performing actions to achieve a goal. The first 11 chapters of this book describe and extend the scope of reinforcement learning. The remaining 11 chapters show that there is already wide usage in numerous fields. Reinforcement learning can tackle control tasks that are too complex for traditional, hand-designed, non-learning controllers. As learning computers can deal with technical complexities, the tasks of human operators remain to specify goals on increasingly higher levels. This book shows that reinforcement learning is a very dynamic area in terms of theory and applications and it shall stimulate and encourage new research in this field.

\title{
How to reference
}

In order to correctly reference this scholarly work, feel free to copy and paste the following:

Cheng-Jian Lin (2008). Reinforcement Evolutionary Learning for Neuro-Fuzzy Controller Design,

Reinforcement Learning, Cornelius Weber, Mark Elshaw and Norbert Michael Mayer (Ed.), ISBN: 978-3902613-14-1, InTech, Available from:

http://www.intechopen.com/books/reinforcement_learning/reinforcement_evolutionary_learning_for_neurofuzzy_controller_design

\section{INTECH}

open science | open minds

\author{
InTech Europe \\ University Campus STeP Ri \\ Slavka Krautzeka 83/A \\ 51000 Rijeka, Croatia \\ Phone: +385 (51) 770447 \\ Fax: +385 (51) 686166 \\ www.intechopen.com
}

\author{
InTech China \\ Unit 405, Office Block, Hotel Equatorial Shanghai \\ No.65, Yan An Road (West), Shanghai, 200040, China \\ 中国上海市延安西路65号上海国际贵都大饭店办公楼405单元 \\ Phone: +86-21-62489820 \\ Fax: +86-21-62489821
}


(C) 2008 The Author(s). Licensee IntechOpen. This chapter is distributed under the terms of the Creative Commons Attribution-NonCommercialShareAlike-3.0 License, which permits use, distribution and reproduction for non-commercial purposes, provided the original is properly cited and derivative works building on this content are distributed under the same license. 